\title{
Effects of lactoferrin on osteogenic differentiation and related gene expressions of osteoblast precursor cells MC3T3-E1 under mechanical strain
}

\author{
Jianfeng Sun ${ }^{1}$, Xiaomeng Zhu ${ }^{1}$, Mengke Ge ${ }^{1}$, Chenchen Zhou ${ }^{2}$, Yuyu $\mathrm{Li}^{2 *}$ \\ 1. Department of Orthodontics, Ningbo Stomatology Hospital, China \\ 2. State Key Laboratory of Oral Diseases and Department of Orthodontics, West China Hospital of \\ Stomatology, Sichuan University, China
}

\begin{abstract}
We aimed to evaluate the effects of lactoferrin $(L F)$ on the osteogenic differentiation and related gene expressions of mouse embryonic osteoblast precursor cells MC3T3-E1 under mechanical strain. MC3T3-E1 cells were randomly divided into control, strain loading, $L F$ and strain loading + LF groups. Alkaline phosphatase (ALP) activity was measured. Cytoskeletal morphology was measured by rhodamine-phalloidin staining. Formation of mineralized nodules was observed by alizarin red staining. The expressions of differentiation-related genes type I collagen (COL-1), interleukin-6 (IL-6) and osteocalcin (OCN) were determined by RT-PCR, and those of p-Runx2 and p-ERK1/2 proteins were detected by Western blotting. The number of ALP positive cells and expressions of OCN, $C O L-1$ and IL-6 were significantly elevated $(P<0.05)$. The optical density of strain loading + LF group was higher than those in strain loading and LF groups after incubation for 4 and 7 days $(P<0.05)$. The cell volume and extension range were elevated in strain loading $+L F$ group compared with those in strain loading group. The amount of mineralized nodules in strain loading + LF group was significantly higher than those in strain loading and LF groups, while it was slightly higher in LF group than that in strain loading group. The expressions of p-ERK1/2 and $p$-Runx 2 in strain loading $+L F$ group exceeded those in strain loading and LF groups $(P<0.05)$. The synergistic action of $L F$ and mechanical strain can effectively promote the proliferation, differentiation and mineralization of osteoblasts, probably being associated with the ERK1/2 signaling pathway.
\end{abstract}

Keywords: lactoferrin; mechanical strain; MC3T3-E1; proliferation; differentiation

Received: 26 $6^{\text {th }}$ October 2021; Accepted: $31^{\text {st }}$ December 2021; Published: $9^{\text {th }}$ Jannuary 2022

\section{Introduction}

Suture distraction osteogenesis (SDO) is commonly utilized to ameliorate jaw bone mass and bone morphology in clinical practice (1). Bone remodeling under the effect of mechanical strain is the biological basis for distraction osteogenesis. As a strain-sensitive cell, osteoblasts play a crucial role in the bone remodeling at the suture under mechanical strain. However, relapse after treatment with SDO is common. Thus, how to improve the efficiency of distraction osteogenesis and stabilize treatment effect have become hot spots. The further study on lactoferrin (LF) provides new possibilities for solving this hot issue. At physiological concentrations, LF can

\footnotetext{
* Corresponding author: Yuyu Li, State Key Laboratory of Oral Diseases and Department of Orthodontics, West China Hospital of Stomatology, Sichuan University, China. E-mail: wrapatnilasge@gmx.de
} 
promote bone growth, stimulate osteoblast proliferation and differentiation, and reduce apoptosis (2). In this study, the influences of different LF concentrations and different strain time on osteogenic effect of osteoblasts were explored in the early stage, which revealed that LF had the most obvious effect of promoting osteoblast differentiation at a concentration of $100 \mu \mathrm{g} / \mathrm{mL}$, while the effect of mechanical strain loading on osteoblasts to promote bone formation reached a peak at $12 \mathrm{~h}$. However, whether LF has biological behavior to promote osteogenic differentiation under mechanical strain loading and its signaling pathways have not been elucidated clearly.

The extracellular signal-regulated kinase $1 / 2$ (ERK1/2) signaling pathway is one of the subfamilies of mitogen-activated protein kinase (MAPK) signaling pathway, and the MAPK signaling pathway is closely related to osteogenic differentiation, which can activate and phosphorylate Runx2 (3), thereby promoting the expression of mineralization-related genes such as osteocalcin (OCN), alkaline phosphatase (ALP) and type I collagen (COL-1). LF can induce the phosphorylation of ERK1/2 in osteoblast-like cells (4). Besides, the phosphorylation and activation of ERK1/2 pathway can enhance the proliferation and survival of osteoblast-like cells (5). Mechanical strain stimulation can also activate the human osteoblast cell line SV-HFO ERK1/2 pathway, and the degree of activity of the ERK1/2 pathway is related to the specific stage of cellular osteogenic differentiation (6). Mechanical strain stimulation is one of the critical factors that regulate the biological response of osteoblasts and maintain the balance of bone tissue remodeling under strain. Alveolar bone remodeling under strain is the biological basis of clinical orthodontic treatment, which moves orthodontic teeth and maintains the balance of bone tissue remodeling under strain through the adjustment of osteoblast or osteoclast function
(7). The proliferative activity of osteoblasts, a kind of strain-sensitive cell, changes after mechanical strain, which directly reflects the state of bone suture tissue remodeling under the action of orthopedic force (8). An in vitro study exhibited that LF is able to promote bone growth and can effectively enhance the proliferative activity of osteoblasts at physiological concentrations (9).

Mouse embryonic osteoblast precursor cells (MC3T3-E1) belong to the cloned cell line of mouse skull and have the features of directed osteogenic differentiation, which are ideal cell lines for exploring biological behaviors such as proliferation and differentiation of osteoblasts. Herein, therefore, MC3T3-E1 osteoblast-like cells were cultured in vitro to observe the effects of LF combined with mechanical strain on osteogenic differentiation and related gene expressions of MC3T3-E1 and the ERK1/2 signaling pathway.

\section{Materials and Methods}

\section{Cells, reagents and apparatus}

Osteoblast cell line MC3T3-E1 was purchased from ATCC (USA), and LF powders (purity $>85 \%$ ) were provided by Sigma-Aldrich (USA). About $10 \mathrm{mg}$ of LF powders were added into $10 \mathrm{~mL} \alpha$-minimum essential medium ( $\alpha$-MEM) containing $10 \%$ fetal bovine serum (FBS) and fully mixed to prepare the LF solution $(1000 \mu \mathrm{g} /$ $\mathrm{mL}$ ). Afterwards, $1 \mathrm{~mL}$ was extracted from the above solution and added into a $9 \mathrm{~mL}$ medium the same as before to prepare a LF solution (10 $\mathrm{mL}, 100 \mu \mathrm{g} / \mathrm{mL}$ ).

The apparatus and reagents included Flexcell-FX5000 tension system, cell incubator (Thermo Fisher Scientific, USA), fluorescence quantitative polymerase chain reaction (PCR) system (ABI StepOne 7300, USA), protein electrophoresis instrument (BioRad, USA), enzyme-linked immunoassay detector (BioTek, 
USA), $\alpha$-MEM (Hyclone, USA), FBS (Gibco, Australia), $0.25 \%$ trypsin, penicillin, streptomycin, RIPA lysate, BCA protein quantification kit, ALP assay kit (Beyotime Institute of Biotechnology Co., Ltd., China), L-ascorbic acid, $\beta$-glycerophosphate sodium (Sigma-Aldrich, USA), TRIzol, reverse transcription kit, and SYBR fluorescence quantitative kit (Takara Bio Inc., Japan).

\section{Cell culture and experimental grouping}

After thawing, MC3T3-E1 cells were routinely cultured in $\alpha$-MEM (containing 10\% FBS +100 $\mathrm{U} / \mathrm{mL}$ penicillin $+0.1 \mathrm{mg} / \mathrm{mL}$ streptomycin) in an incubator at $37^{\circ} \mathrm{C}$ with $5 \% \mathrm{CO}_{2}$. The medium was changed every 3 days and cells were passaged when $70-80 \%$ confluence was reached. The synchronously-growing cells were randomly divided into 4 groups, with 6 wells in each group: a) control group, b) strain loading group: $12 \%$ mechanical strain for $24 \mathrm{~h}, \mathrm{c})$ LF group: pre-incubation with LF at a final concentration of $100 \mu \mathrm{g} / \mathrm{mL}$ for $30 \mathrm{~min}$, and d) strain loading + LF group: pre-incubation with LF $(100 \mu \mathrm{g} / \mathrm{mL})$ for $30 \mathrm{~min}$, plus $12 \%$ mechanical strain loading for $24 \mathrm{~h}$.

Flexcell-FX5000 tension system was controlled by a computer. Under the control of the host, the sealed valve device in the pressure transmitter automatically adjusted and maintained the pressure. It had six circular holes, each of which had a matched piston and fixed table. The hollow circular bubble device can keep the sample in the middle of each hole. In this study, the static waveform was set for pressing.

\section{Measurement of ALP activity}

MC3T3-E1 cells were inoculated into a 24-well plate at a density of $1 \times 10^{3}-1 \times 10^{4}$ cells/well. The medium in each well was about $500 \mu \mathrm{L}$. After cells adhered to the wall, phosphate-buffered saline (PBS) was immediately used for washing and induction medium was adopted. The cells were cultured in an incubator with $5 \% \mathrm{CO}_{2}$ at $37^{\circ} \mathrm{C}$, and the culture medium was replaced in time during the incubation. After 7 days of induction, the culture medium was carefully aspirated and discarded, and then the cells were treated according to different conditions. The medium during the treatment was still the induction medium. After that, the cells were washed with PBS twice to three times, stained according to the instructions of ALP assay kit, observed under a microscope, and recorded through photography.

\section{Detection of cell proliferation by cell counting kit-8 (CCK-8) assay}

MC3T3-E1 cells in the logarithmic growth phase were inoculated into a 96-well plate at a density of $3 \times 10^{7} / \mathrm{L}, 10 \mu \mathrm{L}$ per well, and 5 replicate wells were set. The cells were cultured in an incubator at $37^{\circ} \mathrm{C}$ with $5 \% \mathrm{CO}_{2}$. Then the cells were treated according to different conditions. On the 1st, 4 th and 7 th days, $100 \mu \mathrm{L}$ of a mixture of CCK-8 and culture medium (v:v, 1:9) was added. The absorbance of each well was measured at 450 $\mathrm{nm}$ on a microplate reader.

\section{Rhodamine-phalloidin staining}

The cells were inoculated into a 24-well plate at a density of $3 \times 10^{7} / \mathrm{L}$ to prepare coverslips, with $1 \mathrm{~mL}$ of cell suspension in each well, and allowed to attach overnight. The cells were treated according to different conditions, and three replicate wells were set. After incubation for 24 $\mathrm{h}$, the culture medium was aspirated and discarded. After washing with pre-heated PBS $\left(37^{\circ} \mathrm{C}\right)$ three times, the cells were fixed by $4 \%$ paraformaldehyde for $10 \mathrm{~min}$. Then they were washed with PBS three times (10 min/time). Next, the cells on coverslips were covered with $200 \mu \mathrm{L}$ of rhodamine-phalloidin $(100 \mathrm{nmol} / \mathrm{L})$, incubated at room temperature in the dark for $30 \mathrm{~min}$, and washed with PBS three times ( $5 \mathrm{~min} /$ time). Finally, the cytoskeletal morphology was observed 
by laser scanning confocal microscope after anti-fluorescence quenching mounting.

\section{Alizarin red staining}

MC3T3-E1 cells were inoculated into a 24-well cell culture plate at a density of $1 \times 10^{3}-1 \times 10^{4}$ cells/well. The medium in each well was about $500 \mu \mathrm{L}$. After cells adhered to the wall, PBS was immediately used for washing and induction medium was adopted. The cells were cultured in an incubator in $5 \% \mathrm{CO}_{2}$ at $37^{\circ} \mathrm{C}$, and the culture medium was replaced in time during the incubation. After 7 days of induction, the culture medium was discarded, and then the cells were treated according to different conditions. The medium during the treatment was still the induction medium to the mineralized stage. After that, the cells were washed with PBS twice to three times, fixed by paraformaldehyde for 5 min. The newly prepared alizarin red dye was dripped into the cell samples (about $1 \mathrm{~mL}$ per well) for staining at $37^{\circ} \mathrm{C}$ for $30 \mathrm{~min}$. Then the samples were washed with double distilled water twice to three times, dried, finally observed and recorded through photography.

\section{RT-PCR}

The experimental cells were digested with 2.5 $\mathrm{g} / \mathrm{L}$ trypsin, centrifuged at $800 \mathrm{rpm}$ for $6 \mathrm{~min}$, and washed with PBS. After centrifugation again, the cells were harvested to extract total ribonucleic acid (RNA) according to the TRIzol reagent instructions. Next, the collected cells were added with $1 \mathrm{~mL}$ TRIzol reagent for reaction at room temperature for $5 \mathrm{~min}$. After that, the cells were added with $0.2 \mathrm{~mL}$ of chloroform, fully mixed, incubated at room temperature for 2-3 $\mathrm{min}$, and centrifuged at 12,000 rpm for 15 $\min$ at $4^{\circ} \mathrm{C}$. Subsequently, aqueous phase was collected and transferred into a new centrifuge tube. The aqueous phase was mixed with isopropanol to precipitate the RNA, and $0.5 \mathrm{~mL}$ of isopropanol was added to each $1 \mathrm{~mL}$ of TRIzol reagent homogenized samples. The samples were fully mixed and incubated at room temperature for $10 \mathrm{~min}$, centrifuged at $12,000 \mathrm{rpm}$ for $10 \mathrm{~min}$ at $4{ }^{\circ} \mathrm{C}$, and washed with $75 \%$ ethanol, followed by centrifugation at 7,500 rpm for 5 min at $4^{\circ} \mathrm{C}$. RNA was dried naturally, added with sterile water, and stored at $-70^{\circ} \mathrm{C}$ for later use. Then, $2 \mu \mathrm{g}$ of total RNA was reversely transcribed into complementary deoxyribonucleic acid (cDNA) using Revert Aid ${ }^{\mathrm{TM}}$ first strand cDNA synthesis kit. Primers were designed by Primer Premier 5.0 software. Primer sequences: $\beta$-actin: forward, 5'-TGGTGAAGGTCGGTGTGAAC-3'; reverse, 5'-GCTCCTGGAAGATGGTGATGG-3'. COL-1: forward, 5'-CTGGCGGTTCAGGTCCAAT-3'; reverse, 5'-TTCCAGGCAATCCACGGC-3'. OCN: forward, 5'-AAGCAGGAGGGCAATAAGGT-3'; reverse, 5'-AGCTGCTGACCATCCCATAC-3'. IL-6: forward: 5'-ATGAAGTTCCTCTCTGCAAGAGACTT-3'; reverse: 5'-ATCACGGTTTGCCGAGTAGACTCT-3'.Real-time PCR was performed in the system composed of DNA template and all cDNA samples under the following conditions: $94^{\circ} \mathrm{C}$ for $20 \mathrm{~s}$, annealing temperature for $20 \mathrm{~s}$ and $72^{\circ} \mathrm{C}$ for $30 \mathrm{~s}, 40 \mathrm{cy}$ cles in total. The relative quantification of gene expression was calculated based on the comparative $\mathrm{CT}$ value method, and the RNA content of each sample was standardized according to the respective U6 level. The mRNA expression level was expressed as relative fluorescence values.

\section{Western blotting}

The cells were cultured for $24 \mathrm{~h}$, washed and scraped. After that, the samples were centrifuged to discard supernatant, and lysed with $100 \mu \mathrm{L}$ (about 10 times the cell volume) of cell lysate containing protease inhibitors and phosphatase inhibitors on the ice for $30 \mathrm{~min}$ or above. Subsequently, protein samples were sonicated, and centrifuged at $12,000 \mathrm{rpm}$ for $15 \mathrm{~min}$ at $4^{\circ} \mathrm{C}$. Next, the supernatant was collected, a portion 
of which was used for protein concentration determination using BCA kit (Beyotime Institute of Biotechnology Co., Ltd., China), and the remaining supernatant was mixed according to protein solution: $5 \times$ loading buffer solution $=$ $4: 1$. The protein solution was placed in water, boiled for about $10 \mathrm{~min}$ and then centrifuged. Afterwards, the samples were spotted, separated by electrophoresis and transferred onto a membrane. Then immuno-hybridization of p-Runx2 and $\mathrm{p}$-ERK1/2 proteins was performed. Finally, images were developed and collected.

\section{Statistical analysis}

All data were statistically analyzed by SPSS Statistics 19.0 software. The quantitative data were expressed as mean \pm standard deviation. Intergroup comparisons were conducted by one-way analysis of variance, and pairwise comparisons were performed by the independent $t$ test. The numerical data were represented as percentage, and intergroup comparisons were carried out with the $\chi^{2}$ test. $\mathrm{P}<0.05$ was considered statistically significant.

\section{Results}

\section{LF enhanced ALP activity of MC3T3-E1 cells under mechanical strain}

The number of ALP positive cells was significantly increased in LF group and strain loading group compared with that in control group $(\mathrm{P}<0.05)$, while the number of ALP positive cells was significantly elevated in strain loading + LF group compared with that in strain loading group $(\mathrm{P}<0.05)$ (Figure 1). The results revealed that both strain loading and LF can promote the expression of ALP in MC3T3-E1 cells, and LF can facilitate the expression of ALP in MC3T3-E1 cells under mechanical strain.

\section{LF promoted proliferation of MC3T3-E1 cells under mechanical strain}

The results of CCK-8 assay indicated that the OD value of cells was significantly higher in strain loading group, LF group and strain loading + LF group in comparison with that in control group after incubation for 1, 4 and 7 days, suggesting statistically significant differences
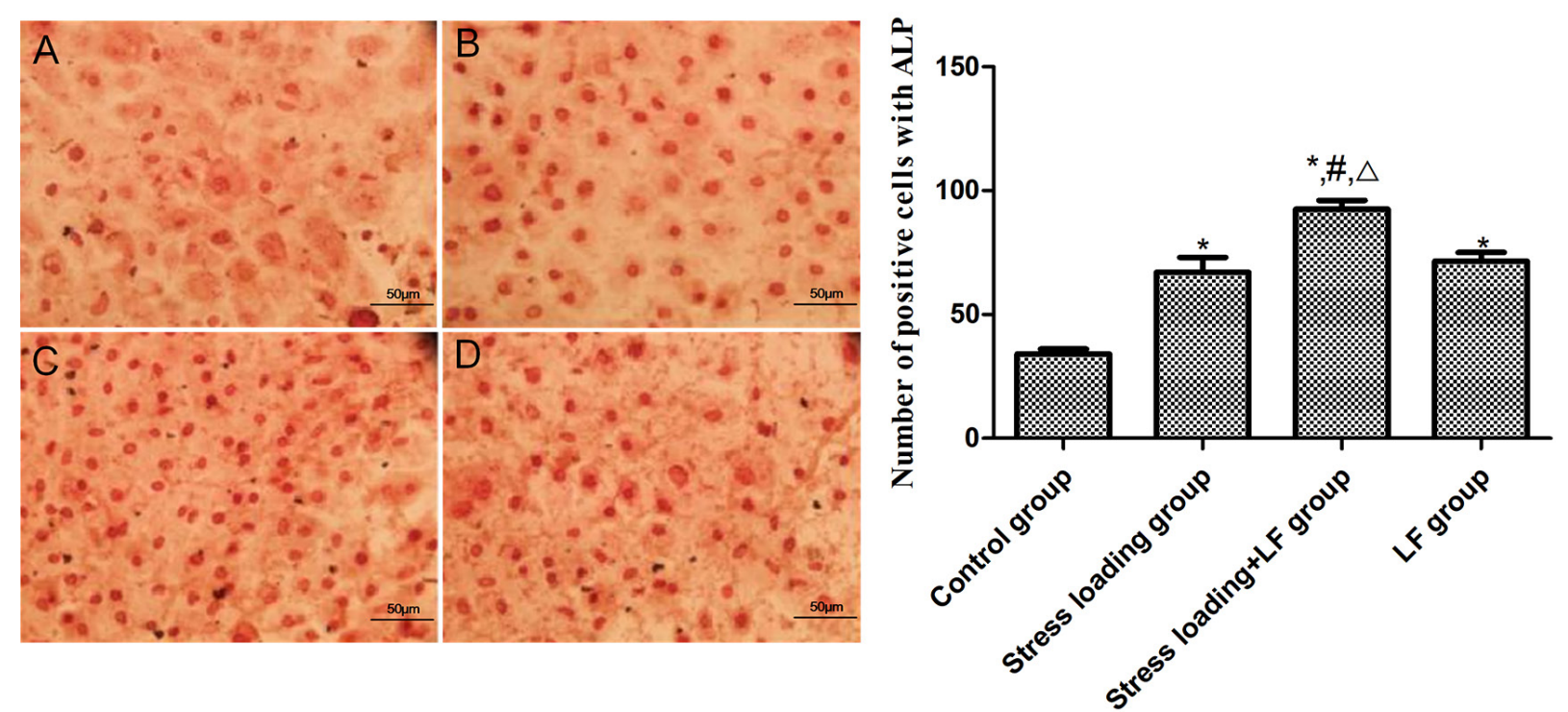

Fig. 1. Effects of LF on ALP activity of MC3T3-E1 cells under mechanical strain. A: Control group; B: strain loading group; C: strain loading + LF group; D: LF group. $x \pm s, n=3$. $* P<0.05$ vs. control group; $\# \mathbf{P}<0.05$ vs. strain loading group; $\Delta \mathrm{P}<0.05$ vs. $\mathbf{L F}$ group. 


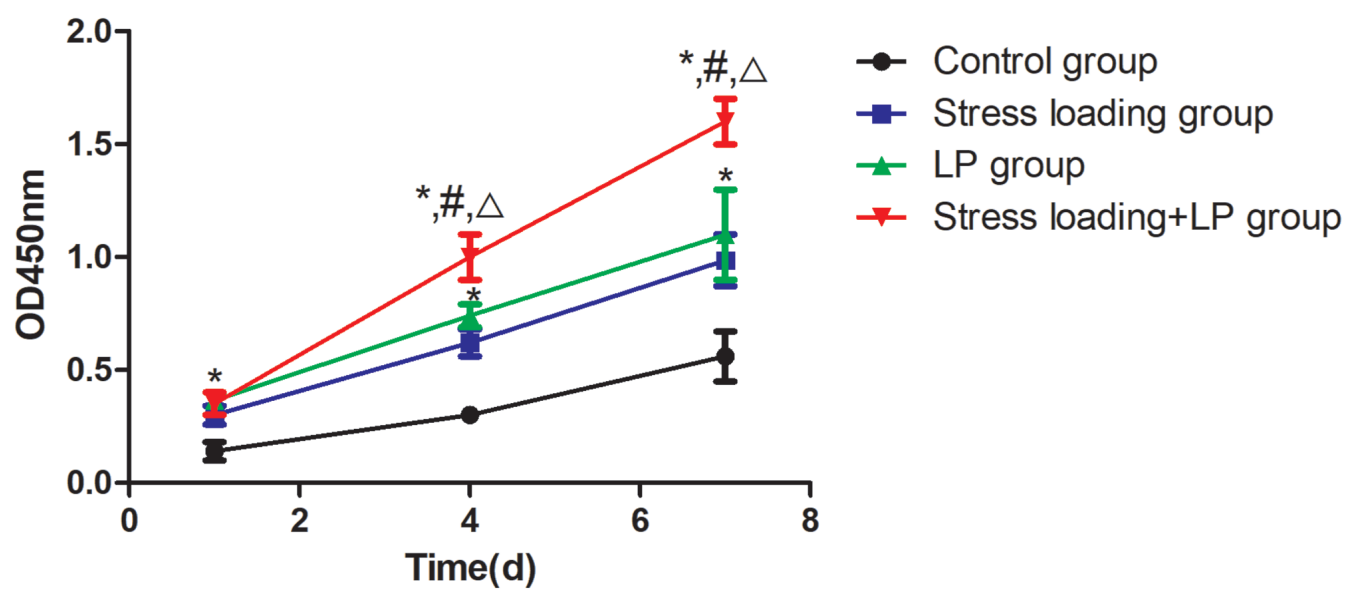

Fig. 2. Effects of LF on proliferation of MC3T3-E1 cells under mechanical strain detected by CCK-8 assay. At the same time point: ${ }^{*} \mathbf{P}<0.05$ vs. control group; $\# \mathbf{P}<0.05$ vs. strain loading group; $\triangle \mathrm{P}<0.05$ vs. LF group.

$(\mathrm{P}<0.05)$. The OD value of cells in strain loading + LF group was higher than that in strain loading group and LF group after incubation for 4 and 7 days, and the differences were statistically significant $(\mathrm{P}<0.05)$ (Figure 2).

\section{Morphological changes of cytoskeleton}

Control group and LP group exhibited the clear direction of fibers and cell internal structure. MC3T3-E1 cells in strain loading group had a small extension and spreading area, and no clear actin fibers and network structure inside the cells were observed. The cell volume and extension range were elevated in strain loading + LF group compared with those in strain loading group (Figure 3).

\section{LF promoted formation of mineralized nodules in MC3T3-E1 cells under mechanical strain} It was confirmed by observation and photographing under the microscope that a few mineralized nodules were formed in control group. The amount of mineralized nodules was significantly increased in LF group and strain loading group compared with that in control group, the degree of mineralized nodules in strain loading + LF group was significantly higher than that in strain loading group and LF group, while it was slightly higher in LF group in comparison with that in strain loading group (Figure 4). The results indicated that LF can promote the mineralization of MC3T3-E1 cells under mechanical strain.

LF increased COL-1 and IL-6 expressions and decreased OCN expression in MC3T3-E1 cells under mechanical strain

The expression levels of OCN, COL-1 and interleukin-6 (IL-6) were significantly increased in strain loading group and LF group compared with those in control group, while the expression levels of OCN, COL-1 and IL-6 were significantly elevated in strain loading + LF group compared with those in strain loading group and LF group, and the differences were statistically significant $(\mathrm{P}<0.05)$ (Figure 5).

LF increased $\mathrm{p}-\mathrm{Runx} 2$ and $\mathrm{p}-\mathrm{ERK} 1 / 2$ expressions in MC3T3-E1 cells under mechanical strain

The expressions of p-ERK1/2 and p-Runx2 were significantly up-regulated in strain loading group and LF group compared with that in control group, and it was significantly higher in strain 

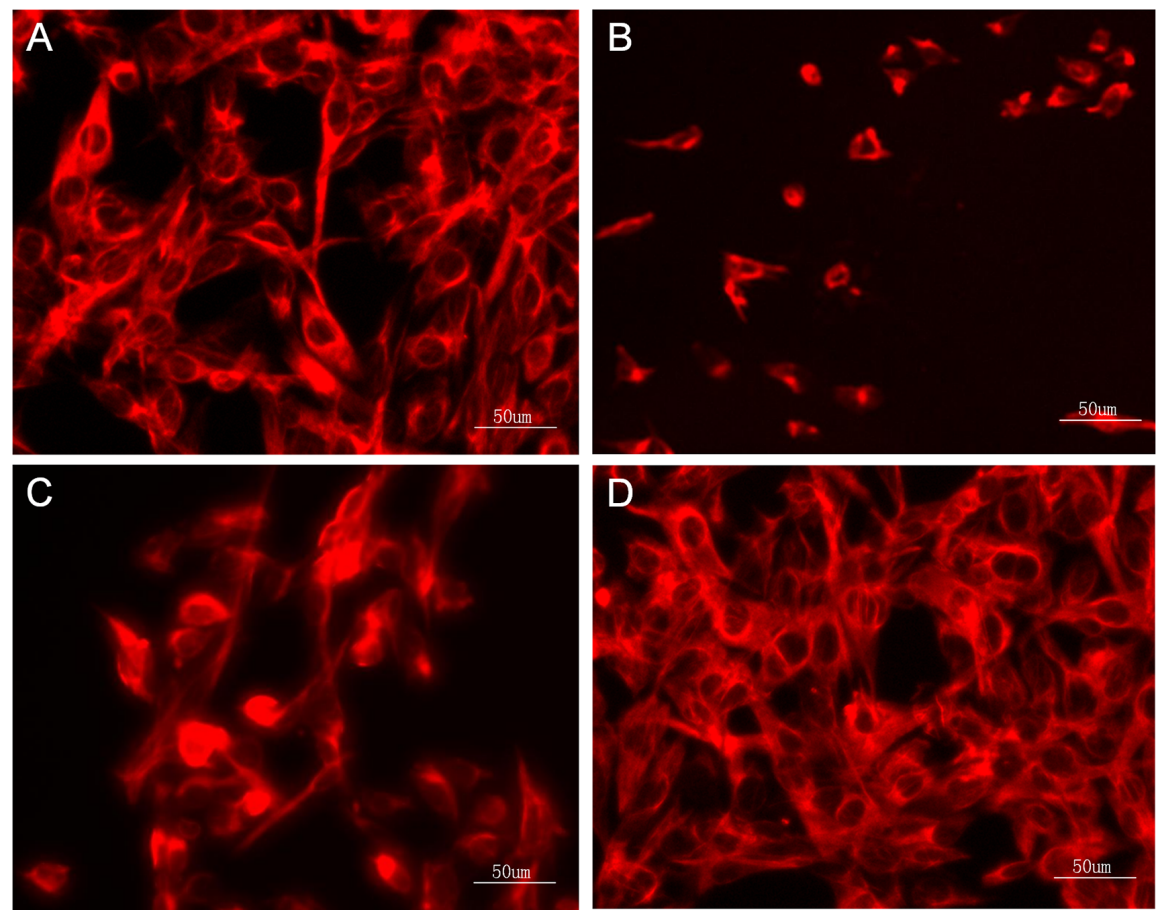

Fig. 3. Effects of LF on morphological changes of MC3T3-E1 cells under mechanical strain. A: Control group; B: strain loading group; C: strain loading + LF group; D: LF group.



Fig. 4. Effects of LF on formation of mineralized nodules in MC3T3-E1 cells under mechanical strain. A: Control group; B: strain loading group; C: strain loading + LF group; D: LF group. 

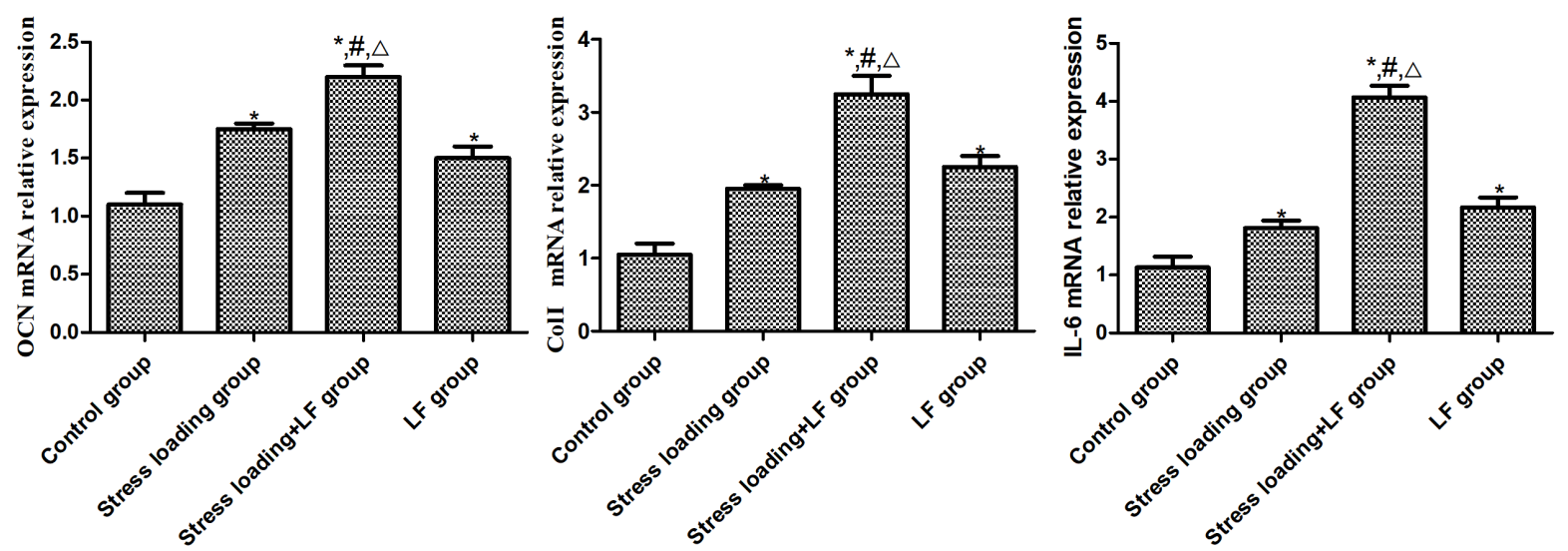

Fig. 5. COL-1, IL-6 and OCN expressions in MC3T3-E1 cells under mechanical strain measured by RTPCR. * $\mathbf{P}<0.05$ vs. control group; \#P<0.05 vs. strain loading group; $\Delta \mathrm{P}<0.05$ vs. $\mathbf{L F}$ group.

loading + LF group in comparison with that in strain loading group and LF group, suggesting statistically significant differences $(\mathrm{P}<0.05)$ (Figure 6).

\section{Discussion}

The results of CCK-8 assay in the present study indicated that the OD value of cells was significantly higher in strain loading group, LF group and strain loading + LF group in comparison with that in control group after incubation for 1 ,
4 and 7 days, and the OD value of cells in strain loading + LF group was higher than that in strain loading group and LF group after incubation for 4 and 7 days. Cytoskeleton is of great significance in the adhesion and extension of osteoblasts on the surface of implants, and the main component of its microfilament is actin (10). This study demonstrated that control group and LP group exhibited the clear direction of fibers and cell internal structure, MC3T3-E1 cells in strain loading group had a small extension and
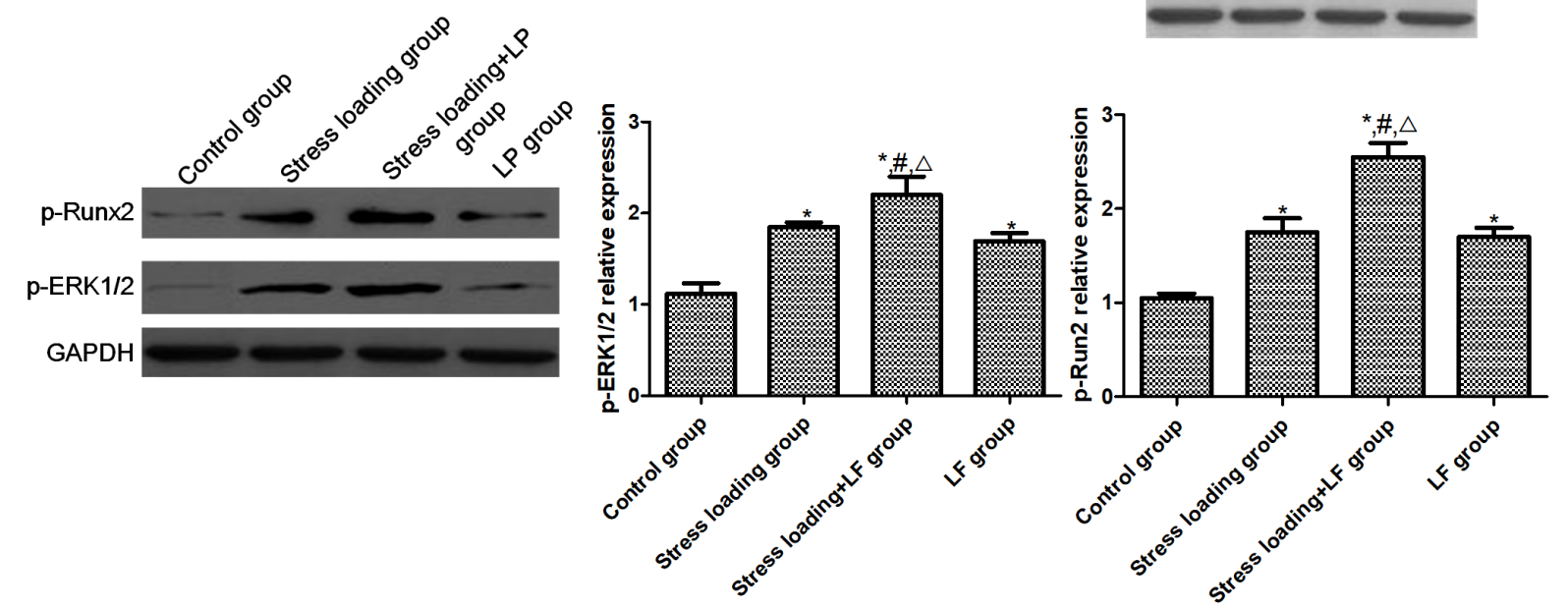

Fig. 6. ERK1/2 and p-ERK1/2 expressions in MC3T3-E1 cells under mechanical strain measured by Western blot. * $\mathbf{P}<0.05$ vs. control group; $\# \mathbf{P}<0.05$ vs. strain loading group; $\triangle \mathrm{P}<0.05$ vs. $\mathrm{LF}$ group. 
spreading area, no clear actin fibers and network structure inside the cells were observed, and the cell volume and extension range were elevated in strain loading + LF group compared with those in strain loading group. Cells development was restricted for the group stimulated by tension. Maybe the applied strain was too high or for too long-time duration.

As an iron-binding glycoprotein, LF exerts its anti-microbial activity, anti-inflammatory, anti-oxidant and iron metabolism functions in the form of iron chelation (11). In a recently published study of Burcel et al the level of lactoferrin measured in the tear film was significantly decreased in the keratoconus and a more severe stage of the keratoconus has been associated with a lower level of lactoferrin (12). A significant positive correlation was found with lactoferrin when compared to corneal hysteresis and the corneal resistance factor.

In the study of Naot et al., LF was injected into the right hemisphere of adult male rats for 5 consecutive days, followed by sacrifice within 24 $\mathrm{h}$ after subcutaneous injection with fluorescent markers, and the results through comparing the skull fragments showed that the bone surface space in LF group increased notably and mineral accumulation rate and bone formation rate elevated remarkably, speculating that LF may enhance the role of osteoblasts and inhibit the function of osteoclasts (13). It was confirmed through the determination of low-density lipoprotein receptor-related protein gene (LRP-1) by Takayama et al. that LF could strengthen the deposition of collagenase, thereby enhancing bone density (14). Cornish reported that LF can stimulate the osteoblast formation cycle and is a potential survival factor for osteoblasts, and LF is also able to reduce the number of osteoclasts, thus inhibiting bone resorption caused by osteoclasts (15).

Inducing the mineralization of osteoblasts is conductive to determining the ability of osteo- genic differentiation and maturation of cells according to the amount of mineralized nodules formed (16). In the previous study, Saos-2 osteoblasts were treated with strain stimulation for $12 \mathrm{~h}$, followed by culture for 14 days, and it was found that calcium nodules formed in cells that received strain stimulation are markedly more than those that did not receive strain stimulation, suggesting that mechanical stimulation may accelerate the mineralization of cells (17). Cornish reported that human recombinant LF (100-1000 $\mu \mathrm{g} / \mathrm{mL}$ ) could prominently induce the formation of mineralized nodules in primary osteoblasts..$^{15}$ The results of this experiment indicated that after osteogenic induction of MC3T3-E1 cells, alizarin red staining showed that cells without special treatment had less mineralized nodule formation, cells after $12 \mathrm{~h}$ of strain had proper formation of mineralized nodules, a marked increase in the formation of mineralized nodules could be observed after cells were added with $100 \mu \mathrm{g} / \mathrm{mL}$ of LF, and cells treated with mechanical strain loading plus $100 \mu \mathrm{g} / \mathrm{ml}$ of LF exhibited a more significant increase in the formation of mineralized nodules. The results were similar to the research results of scholars, and further demonstrated that LF can more effectively promote cell mineralization and maturation after mechanical strain loading, and there is a synergistic effect between them. Meanwhile, the amount of mineralized nodules formed after strain loading only was less than that of LF only, it may be because LF continuously acted on the cells, and strain loading only acted on the cells at the first $12 \mathrm{~h}$, which may continue to weaken as mineralization conducted.

ALP is a sign of early osteoblast differentiation, and OCN represents osteoblast maturation. COL1 is one of the main components that constitute the extracellular matrix of bone tissues, indicating that early strain stimulation can promote the synthesis of extracellular matrix of bone tissues, and it is the material basis of bone tissue remod- 
eling under strain (18). IL-6, as a multifunctional cytokine, participates in the periodontal tissue remodeling during orthodontic tooth movement and acts as an effective osteoclast activating factor to stimulate osteoclast production and bone resorption activities. A previous study revealed that the expressions of cytokines and bioactive substances such as IL-1, IL-6, TNF and RANKL that regulate osteoclasts can be detected during orthodontic tooth movement and periodontitis (19). The present study showed that the number of ALP positive cells and the expression levels of OCN, COL-1 and IL-6 were significantly increased in strain loading group and LF group compared with those in control group, while the number of ALP positive cells and the expression levels of OCN, COL-1 and IL-6 were significantly elevated in strain loading + LF group compared with those in strain loading group and LF group, and the differences were statistically significant $(\mathrm{P}<0.05)$, suggesting that both strain loading and LF can promote the differentiation of MC3T3-E1 cells into mature osteoblasts.

After receiving mechanical stimulation, osteoblasts can convert the mechanical signal into a series of biochemical signals in the cells and reach the site of action through the signal transduction pathway, leading to the changes in gene expression. The ERK1/2 signal pathway is an important signal transduction pathway in the strain signal transduction process of osteoblasts, and some cytokines induced after its activation are indispensable for differentiation, maturation and functional activity of osteoblasts and osteoclasts (20). Multiple studies have confirmed that the ERK1/2 signaling pathway plays an important role in strain-mediated various physiological functions of osteoblasts, and is one of the earliest signal responses after osteoblasts receive mechanical strain (21). ERK is usually located in the cytoplasm. Once activated by mechanical strain, ERK1/2 quickly crosses the nuclear membrane and activates transcription factors, which can further regulate the expression of some important downstream cytokines such as IL-1, IL-6, prostaglandins, alkaline phosphatase, osteocalcin, insulin-like growth factor-I, adenylate cyclase- 2 , transforming growth factor- $\beta$, bone morphogenetic protein and type I collagen. However, the research results are different due to different research objects and differences in strain loading methods. The related signaling pathways by which mechanical strain and LF synchronously act on osteoblasts have not been reported. This tissue was explored for the first time in the present study, but whether the effects of LF and mechanical strain on MC3T3-E1 cells are simultaneously mediated by the ERK $1 / 2$ signaling pathway, and whether they play a synergistic role in activating the ERK1/2 signaling pathway are needed to be further investigated. In addition to the ERK1/2 pathway, the MAPK signaling pathway has multiple subfamilies, which play important roles in the signal transduction after strain stimulation. Several studies have also reported that LF activates the p-38MAPK pathway and promotes osteogenic differentiation. These studies suggest that whether p-38MAPK signaling pathway is also involved when LF and strain stimulation act simultaneously can be studied. The above issues will also provide new ideas to explore the mechanism of adding LF to increase bone formation in the process of SDO.

\section{Conclusion}

In conclusion, the synergistic action of LF and mechanical strain can effectively promote the proliferation, differentiation and mineralization of osteoblasts, and its mechanism may be associated with the ERK1/2 signaling pathway.

\section{Acknowledgements}

This study was financially supported by the National Nature Science Foundation of China (No. 81300905 and 81470777). 


\section{Authors' contributions}

Jianfeng Sun and Yuyu Li designed this study and prepared this manuscript; Xiaomeng Zhu, Mengke Ge and Chenchen Zhou collected and analyzed experimental data. All authors have approved the submission and publication of this manuscript.

\section{Conflicts of interest}

The authors have no conflicts of interest relevant to this article.

\section{References}

1. White N, Evans M, Dover MS, Noons P, Solanki G, Nishikawa H. Posterior calvarial vault expansion using distraction osteogenesis. Childs Nerv Syst. 2009;25(2):231-6. DOI: 10.1007/s00381-008-0758-6

2. Baker EN, Baker HM. A structural framework for understanding the multifunctional character of lactoferrin. Biochimie. 2009;91(1):3-10. DOI: 10.1016/j.biochi.2008.05.006

3. Xiao G, Jiang D, Gopalakrishnan R, Franceschi RT. Fibroblast growth factor 2 induction of the osteocalcin gene requires MAPK activity and phosphorylation of the osteoblast transcription factor, Cbfa1/Runx2. J Biol Chem. 2002;277(39):36181-7. DOI: 10.1074/jbc. M206057200

4. Franceschi RT, Xiao G. Regulation of the osteoblast-specific transcription factor, Runx2: responsiveness to multiple signal transduction pathways. J Cell Biochem. 2003;88(3):446-54. DOI: 10.1002/jcb.10369

5. Nakajima K, Kanno Y, Nakamura M, Gao XD, Kawamura A, Itoh F, et al. Bovine milk lactoferrin induces synthesis of the angiogenic factors VEGF and FGF2 in osteoblasts via the p44/p42 MAP kinase pathway. Biometals. 2011;24(5):847-56. DOI: 10.1007/ s10534-011-9439-0

6. Chang L, Karin M. Mammalian MAP kinase signalling cascades. Nature. 2001;410(6824):37-40. DOI: $10.1038 / 35065000$

7. Veeriah V, Paone R, Chatterjee S, Teti A, Capulli M. Osteoblasts Regulate Angiogenesis in Response to Mechanical Unloading. Calcif Tissue Int. 2019;104(3):34454. DOI: $10.1007 / \mathrm{s} 00223-018-0496-\mathrm{Z}$
8. Ikegame M, Ejiri S, Okamura H. Expression of Non-collagenous Bone Matrix Proteins in Osteoblasts Stimulated by Mechanical Stretching in the Cranial Suture of Neonatal Mice. J Histochem Cytochem. 2019;67(2):107-16. DOI: 10.1369/0022155418793588

9. Kong YY, Du M, Liu M, Zhang LW. Research progress in physio-chemical characteristics and osteogenic activity of lactoferrin. Food Sci. 2012;33:318-22.

10. Jones TJ, Adapala RK, Geldenhuys WJ, Bursley C, AbouAlaiwi WA, Nauli SM, et al. Primary cilia regulates the directional migration and barrier integrity of endothelial cells through the modulation of hsp27 dependent actin cytoskeletal organization. J Cell Physiol. 2012;227(1):70-6. DOI: 10.1002/jcp.22704

11. Singh A, Ahmad N, Varadarajan A, Vikram N, Singh $\mathrm{TP}$, Sharma S, et al. Lactoferrin, a potential iron-chelator as an adjunct treatment for mucormycosis-A comprehensive review. Int J Biol Macromol. 2021;187:98898. DOI: 10.1016/j.ijbiomac.2021.07.156

12. Burcel MG, Constantin M, Ionita G, Dascalescu D, Ionescu C, Stanila D, et al. Levels of lactoferrin, lysozyme and albumin in the tear film of keratoconus patients and their correlations with important parameters of the disease. Rev Romana Med Lab. 2020;28(2):15361. DOI: $10.2478 / \mathrm{rrlm}-2020-0018$

13. Naot D, Grey A, Reid IR, Cornish J. Lactoferrin-a novel bone growth factor. Clin Med Res. 2005;3(2):93-101. DOI: $10.3121 / \mathrm{cmr} .3 .2 .93$

14. Takayama Y, Takezawa T. Lactoferrin promotes collagen gel contractile activity of fibroblasts mediated by lipoprotein receptors. Biochem Cell Biol. 2006;84(3):268-74. DOI: 10.1139/o06-041

15. Cornish J. Lactoferrin promotes bone growth. Biometals. 2004;17(3):331-5. DOI: 10.1023/B:BIOM.0000027713.18694.91

16. Abourehab MA. Hyaluronic acid modified risedronate and teriparatide co-loaded nanocarriers for improved osteogenic differentiation of osteoblasts for the treatment of osteoporosis. Curr Pharm Design. 2019;25(27):2975-88. DOI: $10.2174 / 13816128256661$ 90801140703

17. Mitsui N, Suzuki N, Maeno M, Mayahara K, Yanagisawa $\mathrm{M}$, Otsuka $\mathrm{K}$, et al. Optimal compressive force induces bone formation via increasing bone sialoprotein and prostaglandin $\mathrm{E}(2)$ production appropriately. Life Sci. 2005;77(25):3168-82. DOI: 10.1016/j. lfs.2005.03.037 
18. Li K, Zhuang P, Tao B, Li D, Xing X, Mei X. Ultra-Small Lysozyme-Protected Gold Nanoclusters as Nanomedicines Inducing Osteogenic Differentiation. Int J Nanomed. 2020;15:4705-16. DOI: 10.2147/IJN. S241163

19. Santamaria-Jr M, Bagne L, Zaniboni E, Santamaria MP, Jardini MAN, Felonato M, et al. Diabetes mellitus and periodontitis: Inflammatory response in orthodontic tooth movement. Orthod Craniofac Res. 2020;23(1):27-34. DOI: 10.1111/ocr. 12340
20. Tang H, He Y, Li L, Mao W, Chen X, Ni H, et al. Exosomal MMP2 derived from mature osteoblasts promotes angiogenesis of endothelial cells via VEGF/Erk1/2 signaling pathway. Exp Cell Res. 2019;383(2):111541. DOI: 10.1016/j.yexcr.2019.111541

21. Brandl N, Zemann A, Kaupe I, Marlovits S, Huettinger P, Goldenberg H, et al. Signal transduction and metabolism in chondrocytes is modulated by lactoferrin. Osteoarthritis Cartilage. 2010;18(1):117-25. DOI: 10.1016/j.joca.2009.08.012 